\title{
Finite Element Analysis-Based Approach for Prediction of Aneurysm-Prone Arterial Segments
}

\author{
Viktor Yu. Dolgov ${ }^{1} \cdot$ Kirill Yu. Klyshnikov ${ }^{1} \cdot$ Evgeny A. Ovcharenko $^{1} \cdot$ Tatiana V. Glushkova $^{1} \cdot$ Andrey V. Batranin $^{2}$. \\ Alena S. Agienko ${ }^{3}$. Yuliya A. Kudryavtseva ${ }^{1}$. Arseniy E. Yuzhalin ${ }^{1}{ }^{\circledR} \cdot$ Anton G. Kutikhin $^{1}$
}

Received: 7 September 2017 / Accepted: 19 January 2018 / Published online: 11 July 2018

(c) The Author(s) 2018

\begin{abstract}
Arterial aneurysms represent a significant cause of morbidity and mortality worldwide; therefore, there is a growing need for the rapid and precise tool or method enabling to predict aneurysm emergence. The objective of this study was to develop a computer-aided method for aneurism prediction. Here we utilized a computer-based approach to establish a non-invasive, high-resolution and rapid method for the prediction of aneurysm-prone arterial segments based on combining microcomputed tomography (microCT) scanning with finite element analysis (FEA). We performed a microCT image binarization and designed a computing algorithm for FEA mesh construction, followed by application of gradient mapping and stress modeling to identify thin-walled high-stress areas responsible for the development of aneurysms. The fidelity of our computing algorithm for FEA mesh construction was similar to the commercially available software. The maximum possible error of our approach did not exceed that of either microCT or clinically available multislice computed tomography angiography scanning. Our computational approach revealed thin-walled arterial segments under a high stress, therefore potentially predicting aneurysm-prone sites. Here we demonstrate our approach for the prediction of arterial segments under a high risk of aneurysm occurrence, which should be further validated in pre-clinical models to be translated into the clinical practice.
\end{abstract}

Keywords Aneurysm $\cdot$ Microcomputed tomography $\cdot$ Finite element analysis $\cdot$ Gradient mapping $\cdot$ Stress modeling

JEL Code I10 Health General

\section{Introduction}

An arterial aneurysm is defined as a focal dilation of an arterial wall $[1,2]$. The two most common types of arterial aneurysms are intracranial and aortic aneurysms [1]. Intracranial aneurysms occur in or near the circle of Willis,

Electronic supplementary material The online version of this article (https://doi.org/10.1007/s40846-018-0422-x) contains supplementary material, which is available to authorized users.

Arseniy E. Yuzhalin

yuzhalin@gmail.com

1 Research Institute for Complex Issues of Cardiovascular Diseases, Sosnovy Boulevard 6, Kemerovo, Russia 650002

2 National Research Tomsk Polytechnic University, Lenin Avenue 30, Tomsk, Russia 634050

3 Kemerovo State Medical University, Voroshilova Street 22a, Kemerovo, Russia 650029 an interconnected system of arteries that supply blood to the brain and surrounding structures; aortic aneurysms occur in the aorta, the main artery in the human body, originating from the left ventricle of the heart and extending down to the abdomen [1]. Being initially asymptomatic, aneurysms can rupture, causing a major clinical event. At least one in 20-30 adults carries an asymptomatic intracranial aneurysm, approximately a quarter of which ruptures during the lifetime [3]. The global death rate from aortic aneurysms tends to increase, reaching almost 3 per 100,000 population in 2010 [4]. Thus, there is currently a need for a screening method to define arterial segments at a high risk of aneurysm development. In terms of biomechanics, the predictive algorithm could be similar for both types of arterial aneurysms [1]; however, a computational approach for the prediction of aneurysm-prone sites in the arterial wall is still an unmet clinical need.

The computational modeling of arterial geometry can be considerably complicated by asymptomatic atherosclerotic 
lesions $[5,6]$. Internal mammary artery (IMA) undergoes atherosclerosis extremely rarely $[7,8]$, thus being the only blood vessel suitable for the development and verification of such approach. Here we suggest to use a microcomputed tomography-aided (microCT) imaging of IMA as a template for developing a computer-based model for aneurysm prediction [9]. In this study, we used bovine IMA, since its biomechanical properties are virtually identical to human IMA, therefore being utilized for arterial replacement in clinical practice $[10,11]$.

For the computational modeling of stress we performed a finite element analysis (FEA), a computerized method subdividing an entire object, e.g. arterial wall, into smaller parts called finite elements [12,13]. The simple equations which model finite elements are then assembled into a larger system of equations that models the arterial wall $[12,13]$. Using this approach, we developed a microCT- and FEAbased model for the prediction of arterial segments prone to the development of aneurysms.

\section{Methods}

\subsection{MicroCT Imaging}

We performed microCT scanning of two bovine IMA using TOLMI-150-10, an original microCT scanner designed in National Research Tomsk Polytechnic University [14]. For microCT, we used the following parameters: X-ray tube voltage of $100 \mathrm{kV}, \mathrm{X}$-ray tube current of $5 \mathrm{~mA}$, exposure time of $1 \mathrm{~s}$ per frame, angle step of $0.35^{\circ}$, and voxel size of $67.3 \mu \mathrm{m}$.

\subsection{MicroCT Data Processing}

Images were imported to MATLAB R2015 (MathWorks) following the reduction of a graylevel image to a binary image by Otsu's method [15] as shown in Fig. 1a. This algorithm assumes that the image contains two classes of pixels (foreground pixels and background pixels) and then calculates the optimum threshold separating these two classes so that their intra-class variance is minimal while their interclass variance is maximal [15].

To address potential issues caused by noise, all input images were low pass filtered using the ImageJ software (National Institutes of Health, Bethesda).

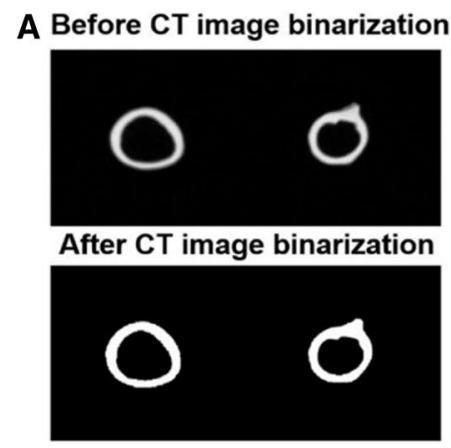

B

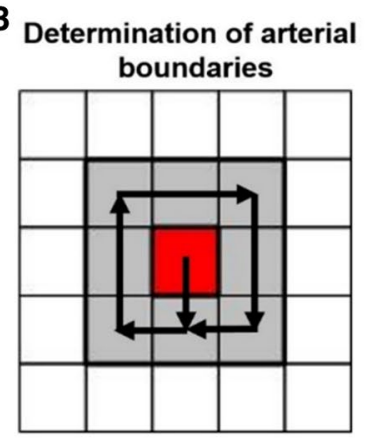

D

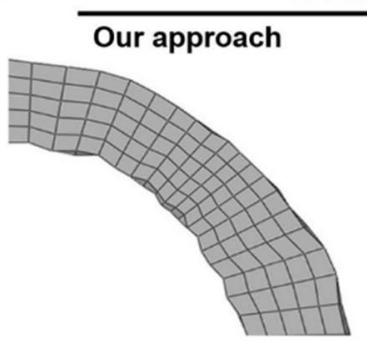

FEA mesh generation

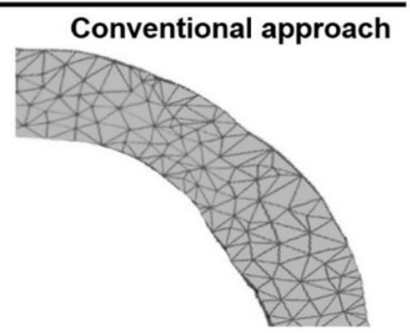

C

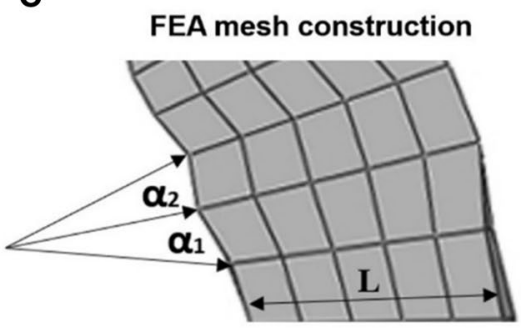

E

Arterial coverage construction

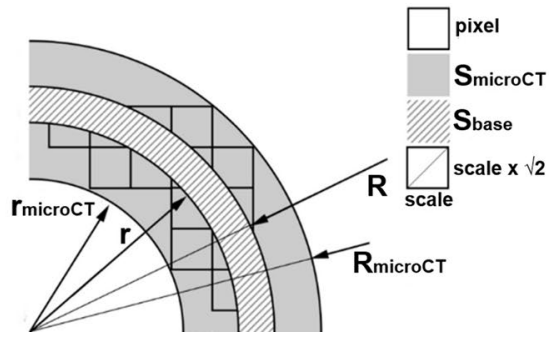

Fig. 1 Microcomputed tomography data processing. a Two representative microcomputed tomography slices before (top) and after (bottom) binarization; b Determination of the bovine internal mammary artery boundaries using Moore neighborhood; c Principle of finite element analysis mesh construction; $\alpha_{1}, \alpha_{2}, \ldots, \alpha_{\mathrm{n}}$ are for the rays, $\mathrm{L}$ is for the thickness. d Finite element analysis mesh generated using our original approach (left) and conventional (right) approach with the angle of $5^{\circ}$ and 5 layers; e Principle of arterial coverage construction for quality control purposes 
Since we found that a conventional approach for FEA mesh generation requires excessive time to complete further stress modeling, we developed a new approach. We first determined the boundaries of IMA in all microCT images using Moore neighborhood [16]. This algorithm worked as shown in Fig. 1b: the selected pixel was bypassed from the bottom up and from left to right on eight neighboring pixels until entering the start pixel for the second time in the same direction it was originally entered [17].

We then calculated the center of mass for each microCT slice and constructed $N$ rays emanating from the center of mass:

$N=$ floor $\left(\frac{360}{\text { angle }}\right)$,

where floor is an integer division rounded down.

Arterial wall thickness was measured by a search of the intersections between the rays and arterial boundaries following the subtraction of the distance between the center of mass and inner boundary from the distance between the center of mass and outer boundary (Fig. 1c). The number of thickness measurements was equal to the number of rays $(N)$ multiplied by the number of images. The number of FEA mesh layers was equal to the ratio of the mean arterial wall thickness to the mean distance between the intersections of the rays with the inner arterial boundary (Fig. 1c).

To complete the construction of FEA mesh nodes, we divided each ray segment between the inner and outer boundary into segments of the same length, number of which was equal to the number of layers (Fig. 1c). For the generation of the FEA mesh, we selected the number of images equal to the ratio of arterial length to the mean distance between the intersections of the rays with the inner arterial boundary. We then compared our original approach for FEA mesh generation to the conventional approach (Amira Software, Zuse Institute Berlin), with a coverage difference of only 3.9\% (Fig. 1d).

The distribution of arterial wall thicknesses was considered as normal according to the Chi square test. Therefore, we calculated minimum and maximum values, mean, and standard deviation. With the aim to better visualize the results, we performed gradient mapping. Each node of the FEA mesh was defined as a certain thickness, and values in the voxels between the nodes depended on values in the nodes. The distribution of thicknesses was represented by a spectrum of colors. As a consensus approach to define which of the areas are considered as thin-walled, we stratified the whole sample of measured thicknesses into the sextiles, with "thin" segments defined as those within a lower sextile $(\leq 0.74 \mathrm{~mm})$.

\subsection{Convergence Analysis}

Convergence analysis was performed to identify the optimal length of the edge of the finite element mesh, whilst maintaining a sufficient level of accuracy of calculations. To do this, the model of a $10 \mathrm{~mm}$ test vessel was divided into finite elements with the following side lengths: 0.14 (number of finite elements $=130680) ; 0.22(48000) ; 0.27$ (21120); 0.34 (12960); 0.40 (7920); 0.44 (3744); 0.48 (3300); 0.54 (2640); and $0.64(1440) \mathrm{mm}$. The finite elements were 20-node quadratic isoparametric elements C3D20. During the analysis, we assessed the maximum and minimum principal stresses of the vascular wall in response to the application of the internal pressure of $140 \mathrm{mmHG}$ on the inner surface of the wall. We found that the stabilization of the solution results occured upon the grid dimension starting from 12960 elements, i.e., with the length of the side of the final element being $0.34 \mathrm{~mm}$, which was used in further experiments (Supplementary Fig. 1).

\subsection{Stress Modeling}

FEA mesh was imported to the Abaqus/CAE (Dassault Systèmes), a software suite for FEA and computer-aided engineering. To define the biomechanical properties of bovine IMA, we examined ten arterial samples in the circumferential direction using a universal testing machine (Zwick/Roell) according to manufacturer's instructions. Briefly, a sample of $5 \mathrm{~mm}$ in length was cut from each vessel and subsequently fixed in custom made clamps specifically designed for this purpose (Supplementary Fig. 2). The specimens were stretched at a traverse speed of $50 \mathrm{~mm} / \mathrm{min}$ and a $50 \mathrm{~N}$ force sensor with an error tolerance of $\pm 1 \%$.

The stress-strain curves generated as a result of physical-mechanical testing were approximated using the seconddegree equation on the basis of the least-squares method to obtain a generalized curve characterizing the biomechanical properties of vessel wall (Supplementary Fig. 3). The resulting polynomial was then imported into Abaqus/CAE software as a "nominal stress-nominal strain" table, on the basis of which, using the built-in tools, an optimal description of the material was selected (Table 1). The candidates for material model were the following: Polynomial model II order, Ogden $\mathrm{N}=3$, and Reduced Polynomial $\mathrm{N}=1$ (Neo Hooke). As a result, it was shown that the Polynomial model II order candidate

Table 1 Coefficients for finite element analysis-based stress modeling obtained using second order polynomial model

\begin{tabular}{lllllll}
\hline $\mathrm{C}_{10}, \mathrm{MPa}$ & $\mathrm{C}_{01}, \mathrm{MPa}$ & $\mathrm{C}_{20}, \mathrm{MPa}$ & $\mathrm{C}_{11}, \mathrm{MPa}$ & $\mathrm{C}_{02}, \mathrm{MPa}$ & $\mathrm{D}_{1}, 1 / \mathrm{MPa}$ & $\mathrm{D}_{2}, 1 / \mathrm{MPa}$ \\
\hline 0.110 & 0.015 & 0.410 & -0.570 & 0.180 & 0.010 & 0.000 \\
\hline
\end{tabular}


demonstrated stability in the entire range of deformations and the best correspondence to the test data plot (Supplementary Fig. 4).

With the aim to identify high stress areas in the arterial wall, we performed a computational modeling using the Abaqus/Standard solver to reveal how IMA reacts to high systolic pressure of $140 \mathrm{mmHg}$ applied to the inner surface of the vessel. The ends of the vessel model were fixed from displacement along the longitudinal axis $(\mathrm{Z})$ and from rotation relative to the central longitudinal axis $(\mathrm{R})$ relative to the cylindrical coordinate system.

Based on the results of convergence analysis, for the modeling we used the C3D20, general-purpose 3D 20-node quadratic isoparametric elements $(3 \times 3 \times 3$ integration points), and von Mises stress, a measure widely applicable for the analysis of plastic deformation of ductile materials. Similar to the definition of thin-walled areas, we divided the whole sample of measured von Mises stress values into the sextiles, with the segments experiencing the highest sextile $(\geq 0.244 \mathrm{MPa})$ defined as at a high stress.

\subsection{Quality Control}

We measured all the distances from the center of mass to either outer or inner boundary of the arterial wall among all microCT slices with the further calculation of the mean distance defined either as $R$ or $r$, respectively (Fig. 1e). The annulus with larger radius $R$ and smaller radius $r$ was defined as Base (Fig. 1e). The figure with the lowest area fully covering the annulus with pixels of scale resolution was annulus with larger radius

$R_{\text {nicro } C T}=R+$ scale $\times \sqrt{2}$

and smaller radius

$r_{\text {micro } C T}=r-$ scale $\times \sqrt{2}$

This was due to the fact that distance between two points of the square with side scale did not exceed

scale $\times \sqrt{2}$

Hence, all squares intersecting the circle were inside the annulus with radii (2) and (3) (Fig. 1e).

We then calculated the maximum possible error of either microCT or multislice computed tomography angiography (MSCTA), a classical CT technique widely used in clinical practice, by the subtraction of the square of virtual annulus limiting the error of either microCT or MSCTA

$$
S_{\text {micro } C T}=\pi \times\left((R+\text { scale } \times \sqrt{2})^{2}-(r-\text { scale } \times \sqrt{2})^{2}\right)
$$

from the square of annulus Base

$S_{\text {base }}=\pi \times\left(R^{2}-r^{2}\right)$

The scale was $0.06731 \mathrm{~mm} /$ pixel and $0.4 \mathrm{~mm} /$ pixel for microCT and MSCTA, respectively.

For the calculation of the actual error, we constructed the coverage Sbase by trapezoidal finite elements. We then drew the triangle with two sides equal to radius $r$ with the angle between them equal to angle, and defined it as $O A B$. Therefore, the formula for calculation of its square was

$S_{O A B}=\frac{1}{2} \times r^{2} \times \sin$ angle

We further drew tangent passing through the $M$, a point of intersection of the bisector of $A B O$ triangle and a circle with center $O$ and radius $R$. We then defined the intersections of rays $O A$ and $O B$ with the tangent passing through the $M$ as $C$ and $D$, respectively. Therefore, the square of triangle $O C D$ was

$S_{O C D}=R^{2} \times \tan \frac{\text { angle }}{2}$,

square of trapezoidal finite element $A B D C$ was $\mathrm{S}_{o c d}-\mathrm{S}_{o a b}$, and

$S_{A B D C}=\left(\left[\frac{360}{\text { angle }}\right]+1\right) \times\left(S_{o c d}-S_{o a b}\right)$

was a minimum coverage of annulus $S_{\text {basic }}$ by our approach.

\section{Results}

\subsection{Distinct Areas of the Arterial Wall Having Different Thicknesses}

We first obtained 1528 images per each of the two bovine IMA using microCT scanning following image binarization and FEA mesh generation. Thicknesses of distinct segments within the arterial wall varied from $<0.7 \mathrm{~mm}$ to $>2 \mathrm{~mm}$, as demonstrated in Fig. 2a. The distribution of arterial wall thicknesses was considered as normal, and mean thickness of the two arteries did not differ significantly (Fig. 2a).

\subsection{High Stress Areas in the Artery Occasionally Match Thin-Walled Regions}

We then questioned whether arterial wall contains high stress areas and whether they correspond to the regions of low thickness. Having performed the examination of bovine IMA in the circumferential direction using a universal testing machine, we identified specific biomechanical properties and further calculated coefficients for the FEA-based stress 
A

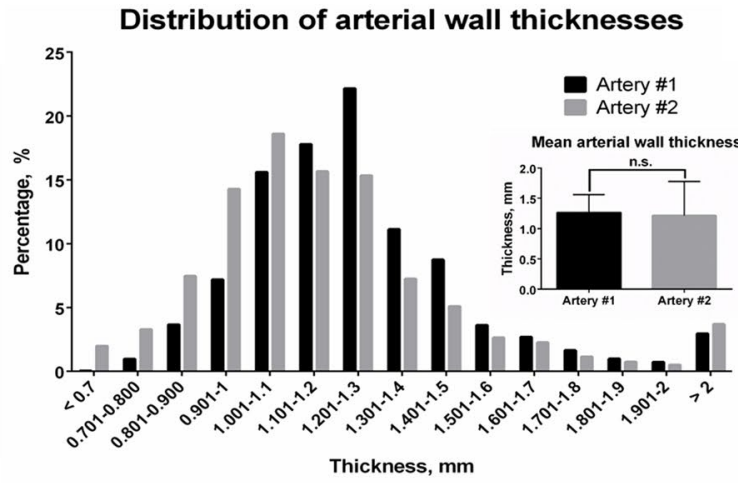

C

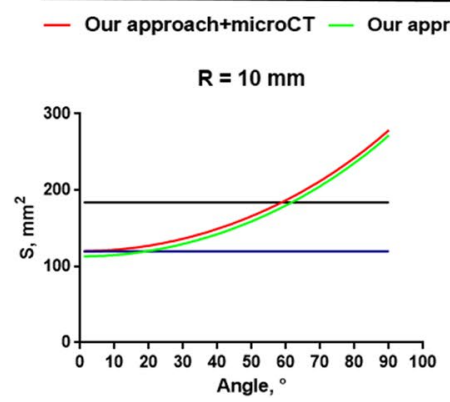

Arterial coverage

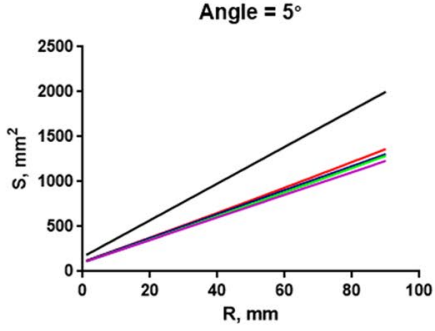

B

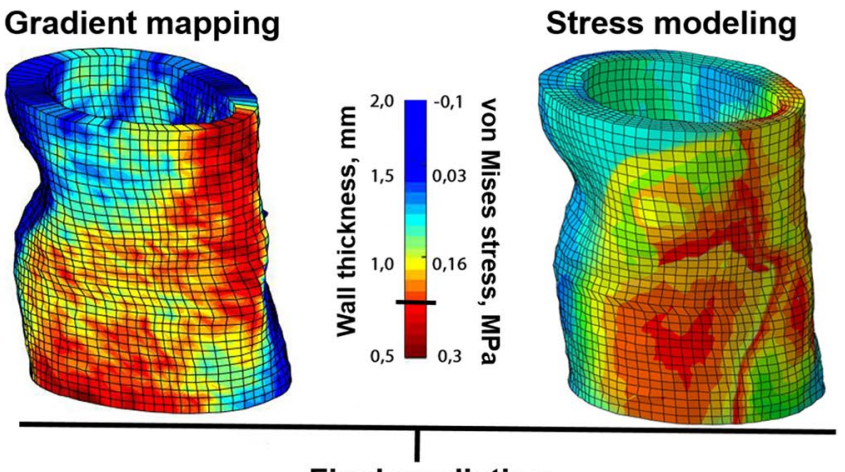

Final prediction

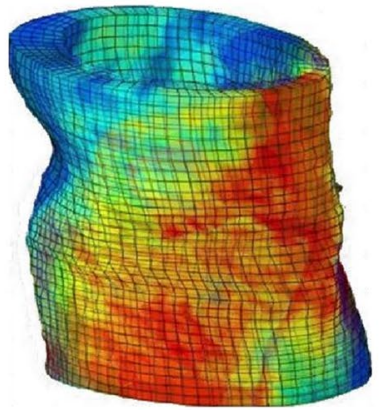

Fig. 2 Performance of the developed approach. a Statistical analysis of the arterial wall thickness; values are represented as mean with a standard deviation, n.s. is for not significant, two-tailed Student's $t$ test. b Comparison of the gradient mapping and stress modeling

modeling (Supplementary Fig. 4 and Table 1). This computational approach revealed areas of high stress in the arterial wall, which were occasionally co-localized with thin-walled areas (Fig. 2b). However, a major proportion of high stress areas did not correspond to the thin areas, and vice versa (Fig. 2b).

\subsection{FEA-Based Approach and Computed Tomography Scanning have Similar Fidelity}

Finally, we evaluated the maximum possible error of our original FEA-based approach. Using the measured radius of $10 \mathrm{~mm}$ and along with the error of microCT $E r r_{\text {microcT }}$,

Err $r_{\text {micro } C T}=S_{\text {micro } C T}-S_{\text {base }}$

it did not exceed the error of both microCT and clinically available MSCTA scanning until the angle was $<7^{\circ}$ or with an angle of $5^{\circ}$ regardless of the radius (Fig. 2c).

\section{Discussion}

Cardiovascular diseases (CVD) are the predominant cause of death globally. One of the most dangerous CVDs is aortic aneurism, pathologically resulting from trauma, results; a black line on the scale indicates the threshold for thinwalled and high stress areas; $\mathbf{c}$ Results of arterial coverage construction

infection or intrinsic abnormality in the elastin and collagen components of the aortic wall. Genetic factors are also playing a role in etiology of aortic aneurisms, with multiple genetic syndromes, such as Marfan, Ehlers-Danlos and others, being associated with their occurrence.

While dormant arterial aneurysms can be asymptomatic, their ruptures cause significant morbidity and mortality [18-21]. Screening is considered as an important tool for reducing risk of ruptures, halving the risk of dying from aneurysms $[18,20]$. Combined screening for intracranial and aortic aneurysms is cost-effective due to their high coprevalence [22]. Despite some promising results demonstrated by the currently available screening techniques, i.e. ultrasonography, CT angiography, and magnetic resonance angiography [18, 20, 23], the search for low-cost, non-invasive, and high-resolution screening methods is still ongoing to further improve efficiency and ultimately reduce the risk of ruptures. In this study we suggest that a widespread diagnostic tool CT can be combined with FEA, gradient mapping, and stress modeling to provide an approach holding all the above-mentioned advantages for a screening of arterial aneurysms. Furthermore, this approach can potentially have an additional edge enabling prediction of arterial segments prone to aneurysm development. 
Here we presented our microCT and FEA-based approach for the prediction of aneurysm-prone arterial segments that included microCT image binarization, FEA mesh construction according to the original algorithm, gradient mapping, and stress modeling. Utilizing this method, we identified that a major proportion of high stress areas did not correspond to the thin areas, and vice versa. We therefore speculated that arterial wall thickness or value of von Mises stress independently are unlikely to be responsible for the predisposition of an arterial segment to aneurysm development; however, both of these parameters determine high-risk sites when combined. Indeed, it is well recognized that thin areas at high stress in the arterial wall are responsible for the development of aneurysms [24-26]. Importantly, we identified these aneurysm-prone arterial segments with a relatively high frequency.

It could be argued that mechanical properties of arterial wall vary between different samples. However, in our study the actual stress values did not reach the critical point and generally corresponded to $3-5 \mathrm{~mm}$ displacement where the results of a tension test were uniform. In addition, the resolution of ultrasonography as well as computed or magnetic resonance tomography angiography were currently quite low $(>625 \mu \mathrm{m})$ whilst the calculation of force-displacement curve is highly operator-dependent all resulting in actual error exceeding that of our computational approach. Because the proposed algorithm demonstrated correct performance and promising results, we speculate that it is not limited to IMA and can be utilized for other arteries.

To sum up, the advantages of our approach are: (1) noninvasiveness; (2) it significantly accelerates stress modeling compared to the conventional algorithm for FEA mesh construction, with a coverage difference of $<4 \%$; (3) its maximum possible error does not exceed the error of either microCT or MSCTA scanning.

Our approach, nonetheless, has limitations. The research presented here is purely a biomechanical study with the aim to develop a computational model for identification of highstress areas in aorta, potentially predicting the formation of aneurysms. For assessment of its clinical relevance, more follow-up work is required to test its feasibility and validity. The next step would be to examine the performance our numerical model in genetically predisposed or chemically induced experimental models of abdominal aortic aneurysms. Alternatively, our approach could be tested in arteries in hydrodynamic bench or pulsating flow setup equipped with micro-ultrasound device. Thus, it is important to note that our model requires validation in pre-clinical models to be considered for use in clinical practice.

Taking together, our approach enables detection of arterial sites predisposed to aneurysm development. Further research should focus on developing a model of arterial wall degeneration to predict not only the areas but also the conditions of aneurysm development.

Acknowledgements This study was supported by the Complex Program of Basic Research under the Siberian Branch of the Russian Academy of Sciences within the Basic Research Topic of Research Institute for Complex Issues of Cardiovascular Diseases No 05462015-0011 "Pathogenetic basis for the development of cardiovascular implants from biocompatible materials using patient-oriented approach, mathematical modeling, tissue engineering, and genomic predictors". The Ethical Committee of the Research Institute for Complex Issues of Cardiovascular Diseases approved the study protocol.

\section{Compliance with ethical standards}

Conflict of interest All authors declare that they have no conflict of interest.

Open Access This article is distributed under the terms of the Creative Commons Attribution 4.0 International License (http://creativecommons.org/licenses/by/4.0/), which permits unrestricted use, distribution, and reproduction in any medium, provided you give appropriate credit to the original author(s) and the source, provide a link to the Creative Commons license, and indicate if changes were made.

\section{References}

1. Humphrey, J. D., \& Taylor, C. A. (2008). Intracranial and abdominal aortic aneurysms: Similarities, differences, and need for a new class of computational models. Annual Review of Biomedical Engineering, 10, 221-246. https://doi.org/10.1146/annurev.bioen g.10.061807.160439.

2. Aggarwal, S., Qamar, A., Sharma, V., \& Sharma, A. (2011). Abdominal aortic aneurysm: A comprehensive review. Experimental \& Clinical Cardiology, 16, 11-15.

3. Korja, M., \& Kaprio, J. (2016). Controversies in epidemiology of intracranial aneurysms and SAH. Nature Reviews Neurology, 12, 50-55. https://doi.org/10.1038/nrneurol.2015.228.

4. Sampson, U. K., Norman, P. E., Fowkes, F. G., Aboyans, V., Yanna, S., Harrell, F. E., Jr., et al. (2014). Global and regional burden of aortic dissection and aneurysms: Mortality trends in 21 world regions, 1990 to 2010. Global Heart, 9(171-180), e10. https://doi.org/10.1016/j.gheart.2013.12.010.

5. Huang, Y., Teng, Z., Sadat, U., Graves, M. J., Bennett, M. R., \& Gillard, J. H. (2014). The influence of computational strategy on prediction of mechanical stress in carotid atherosclerotic plaques: Comparison of 2D structure-only, 3D structure-only, one-way and fully coupled fluid-structure interaction analyses. Journal of Biomechanics, 47, 1465-1471. https://doi.org/10.1016/j.jbiom ech.2014.01.030.

6. Morbiducci, U., Kok, A. M., Kwak, B. R., Stone, P. H., Steinman, D. A., \& Wentzel, J. J. (2016). Atherosclerosis at arterial bifurcations: Evidence for the role of haemodynamics and geometry. Journal of Thrombosis and Haemostasis, 115, 484-492. https:// doi.org/10.1160/TH15-07-0597.

7. Otsuka, F., Yahagi, K., Sakakura, K., \& Virmani, R. (2013). Why is the mammary artery so special and what protects it from atherosclerosis? Annals of Cardiothoracic Surgery, 2, 519-526. https ://doi.org/10.3978/j.issn.2225-319X.2013.07.06.

8. Perrotta, I., Sciangula, A., Concistrè, G., Mazzulla, S., Aquila, S., \& Agnino, A. (2014). Internal mammary artery atherosclerosis: 
An ultrastructural study of two cases. Ultrastructural Pathology, 38, 199-203. https://doi.org/10.3109/01913123.2013.868568.

9. Boerckel, J. D., Mason, D. E., McDermott, A. M., \& Alsberg, E. (2014). Microcomputed tomography: Approaches and applications in bioengineering. Stem Cell Research \& Therapy, 5, 144. https://doi.org/10.1186/scrt534.

10. Barbarash, L. S., Ivanov, S. V., Zhuravleva, I. Iu., Anufriev, A. I., Kazachek, Ia. V., Kudriavtseva, Iu. A., et al. (2006). Twelve-year experience of bioprosthesis implantation into infrainguinal arteries. Angiologiia i sosudistaia khirurgiia, 12, 91-97.

11. Poyrazoglu, H. H., Avşar, M. K., Tor, F., Erdem, S., Göçen, U., \& Bayraktar, I. (2008). Systemic pulmonary shunt performed with Shelhigh internal mammary artery: Early results. Heart Surgery Forum, 11, E50-E53. https://doi.org/10.1532/HSF98.20071139.

12. Ovcharenko, E. A., Klyshnikov, K. U., Vlad, A. R., Sizova, I. N., Kokov, A. N., Nushtaev, D. V., et al. (2014). Computer-aided design of the human aortic root. Computers in Biology and Medicine, 54, 109-115. https://doi.org/10.1016/j.compbiomed .2014.08.023.

13. Ovcharenko, E. A., Klyshnikov, K. U., Yuzhalin, A. E., Savrasov, G. V., Kokov, A. N., Batranin, A. V., et al. (2016). Modeling of transcatheter aortic valve replacement: Patient specific vs general approaches based on finite element analysis. Computers in Biology and Medicine, 69, 29-36. https://doi.org/10.1016/j.compbiomed .2015.12.001.

14. Batranin, A. V., Chakhlov, S. V., Grinev, D. V., Kapranov, B. I., \& Klimenov, V. A. (2013). Design of the X-ray micro-CT scanner TOLMI-150-10 and its perspective application in non-destructive evaluation. Applied Mechanics and Materials, 379, 3-10. https:// doi.org/10.4028/www.scientific.net/AMM.379.3.

15. Otsu, N. (1979). A threshold selection method from gray-level histograms. IEEE Transactions on Systems, Man, and Cybernetics, 9, 62-66. https://doi.org/10.1109/TSMC.1979.4310076.

16. Sharma, P., Diwakar, M., \& Lal, N. (2013). Edge detection using Moore neighborhood. International Journal of Computer Applications, 61, 26-30. https://doi.org/10.5120/9910-4506.

17. Rajashekar Reddy, P., Amarnadh, V., \& Bhaskar, M. (2012). Evaluation of stopping criterion in contour tracing algorithms. International Journal of Computer Science and Information Technology, 3, 3888-3894.
18. Ali, M. U., Fitzpatrick-Lewis, D., Miller, J., Warren, R., Kenny, M., Sherifali, D., et al. (2016). Screening for abdominal aortic aneurysm in asymptomatic adults. Journal of Vascular Surgery, 64, 855-1868. https://doi.org/10.1016/j.jvs.2016.05.101.

19. Davis, F. M., Rateri, D. L., \& Daugherty, A. (2015). Abdominal aortic aneurysm: Novel mechanisms and therapies. Current Opinion in Cardiology, 30, 566-573. https://doi.org/10.1097/ HCO.0000000000000216.

20. Nasr, D. M., \& Brown, R. D., Jr. (2016). Management of unruptured intracranial aneurysms. Current Cardiology Reports, 18, 86. https://doi.org/10.1007/s11886-016-0763-4.

21. Etminan, N., \& Rinkel, G. J. (2016). Unruptured intracranial aneurysms: Development, rupture and preventive management. Nature Reviews Neurology, 12, 699-713. https://doi.org/10.1038/nrneu rol.2016.150.

22. Ball, B. Z., Jiang, B., Mehndiratta, P., Stukenborg, G. J., Upchurch, G. R., Jr., Meschia, J. F., et al. (2016). Screening individuals with intracranial aneurysms for abdominal aortic aneurysms is cost-effective based on estimated coprevalence. Journal of Vascular Surgery, 64(811-818), e3. https://doi.org/10.1016/j. jvs.2016.05.065.

23. Hahn, B., Bonhomme, K., Finnie, J., Adwar, S., Lesser, M., \& Hirschorn, D. (2016). Does a normal screening ultrasound of the abdominal aorta reduce the likelihood of rupture in emergency department patients? Clinical Imaging, 40, 398-401. https://doi. org/10.1016/j.clinimag.2015.11.021.

24. Jeong, W., \& Rhee, K. (2012). Hemodynamics of cerebral aneurysms: Computational analyses of aneurysm progress and treatment. Computational and Mathematical Methods in Medicine, 2012, 782801. https://doi.org/10.1155/2012/782801.

25. Meng, H., Tutino, V. M., Xiang, J., \& Siddiqui, A. (2014). High WSS or low WSS? Complex interactions of hemodynamics with intracranial aneurysm initiation, growth, and rupture: Toward a unifying hypothesis. American Journal of Neuroradiology, 35, 1254-1262. https://doi.org/10.3174/ajnr.A3558.

26. Simsek, F. G., \& Kwon, Y. W. (2015). Investigation of material modeling in fluid-structure interaction analysis of an idealized three-layered abdominal aorta: Aneurysm initiation and fully developed aneurysms. Journal of Biological Physics, 41, 173201. https://doi.org/10.1007/s10867-014-9372-x. 